\title{
Degenerate fermion gas heating by hole creation
}

\author{
Eddy Timmermans \\ Theoretical Division (T-4), Los Alamos National Laboratory, Los Alamos, NM 87545
}

\begin{abstract}
Loss processes that remove particles from an atom trap leave holes behind in the single particle distribution if the trapped gas is a degenerate fermion system. The appearance of holes increases the temperature and we show that the heating is (i) significant if the initial temperature is well below the Fermi temperature $T_{F}$, and (ii) increases the temperature to $T \geq T_{F} / 4$ after half of the system's lifetime, regardless of the initial temperature. The hole heating has important consequences for the prospect of observing Cooper-pairing in atom traps.
\end{abstract}

PACS numbers(s):03.75.Fi, 05.30.Jp, 32.80Pj, 67.90.+z 
Insofar as the ultra-cold atoms behave as an equilibrium system, the neutral atom trap provides a laboratory for the study of low-temperature phenomena. Recent experiments with atomic Bose-Einstein condensates (BECs) [1] further highlight this connection by reporting genuine superfluid behavior [2]. Similarly, the observation of fermion pairing in the gas phase would be of equal, or even greater interest [3], and the quest for atom-trap fermion superfluidity has begun in earnest. In this Letter, we discuss the effects of particle-loss to which the metastable ultra-cold atom gases are prone. In addition to limiting the system's lifetime, $\tau_{L}$, the loss processes leave behind holes in the single particle distribution of a degenerate fermion gas (of temperature $T$ significantly lower than the Fermi-temperature $\left.T_{F}\right)$. The fermions heat in the subsequent relaxation and, without additional cooling, the system's temperature doubles from its initial value $T_{0}$ within a time $\tau_{2} \sim 20\left[T_{0} / T_{F}\right]^{2} \tau_{L}$. After $t \sim 0.5 \tau_{L}$ the fermion gas heats up to $T \geq 0.25 T_{F}$, regardless of the initial temperature. The competition of superfluid formation with 'Fermi-hole heating' leads to stringent lowerbounds for the strength of the inter-atomic attraction that would pair the fermions.

Introduction In atom traps, inelastic collisions impart kinetic energy to the scattering products, which subsequently leave the trap. Scattering with background atoms and inelastic two-and three-body collisions decrease the local particle density, $n$, as $\dot{n}=-\gamma n, \dot{n}=$ $-\beta n^{2}, \dot{n}=-\alpha n^{3}$, respectively. Although two and three-body scattering is suppressed in a single fermion gas, so are the inter-atomic interactions that relax the system to thermal equilibrium. By trapping ${ }^{40} K$-atoms in two different internal states D. Jin's group circumvented the problem and succeeded in cooling fermion atoms evaporatively (i.e. by removing the highest energy atoms) to degeneracy 《4. Most recently, R. Hulet's group at Rice University [5] and C. Salomon's group at the ENS, Paris [6], cooled fermion atoms $\left({ }^{6} \mathrm{Li}\right)$ by bringing them in thermal contact with ultra-cold $\left({ }^{7} \mathrm{Li}\right)$-bosons. The lowest reported temperatures [5] are $T \sim 0.25 T_{F}$, and the fermion mixture cooling efforts appear to have encountered a limit in this temperature range [4]. Calculations suggest that Pauli-blocking plays a role [7] but this effect alone does not seem to explain the limit. An alternative motivation for creating fermion gas mixtures is the prospect of s-wave Cooper-pairing. This scheme - 
the most likely mechanism to achieve fermion superfluidity at realistic atom trap densities, $n \sim 10^{12}-10^{15} \mathrm{~cm}^{-3}$ - requires the s-wave interaction between two types of distinguishable atoms to be attractive (i.e. described by a negative scattering length, $a<0$ ). Whether the atom mixtures are created to equilibrate the system or to form Cooper-pairs, the unlike fermion interactions introduce loss processes.

We consider a weakly interacting gas mixture of the same fermion isotope in two different internal states, 1 and 2. The optimal mixture contains equal particle densities $n_{1}=n_{2}=n$, corresponding to equal particle numbers, $N_{1}=N_{2}=N$ [ 8 . The usual trapped neutral atom gases are dilute in the sense that $|a| / r<<1$ where $r$ denotes the average inter-particle distance, $r=n^{-1 / 3}$ (typically, $|a| \sim 1 \mathrm{~nm}$ whereas $r \sim 1 .-0.1 \mu \mathrm{m}$ ). In contrast, the quest for fermion superfluidity necessitates an unusually strong neutral atom attraction. This condition follows from the critical temperature $T_{c}$ for s-wave Cooper-pairing: in the described homogeneous fermion mixture of Fermi-momentum $k_{F}=\left(6 \pi^{2}\right)^{1 / 3} r^{-1} \approx 3.9 r^{-1}$, Fermi-energy $\epsilon_{F}=\hbar^{2} k_{F}^{2} / 2 m$, and Fermi-temperature $T_{F}=\epsilon_{F} / k_{B}$ (where $k_{B}$ is the Boltzmann constant) $T_{c}$ equals [9]

$$
T_{c} \approx 0.3 T_{F} \exp ^{-\frac{\pi}{2 k_{F}|a|}}=0.3 T_{F} \exp ^{-0.4 \frac{r}{|a|}}
$$

With $T_{F} \approx 3.65 \mu \mathrm{K} A^{-1}\left(n / n_{0}\right)^{2 / 3}$, where $n_{0}$ is a realistic reference density, $n_{0}=10^{12} \mathrm{~cm}^{-3}$, and $A$ the mass number, we find that the prefactor of (1) is accessible to present day atom trap technology, but with $|a| / r \sim 10^{-3}, T_{c} \approx 0.3 T_{F} \exp (-400)$.

Lifetime restrictions Even if such exponentially low temperatures can be reached, the time scale on which the superfluid forms, $\tau_{\text {form }}$, exceeds any realistic lifetime $\tau_{L}$. This formation time, $\tau_{\text {form }}$, depends on the Fermi-time scale, $\tau_{F e r m i}=\hbar / \epsilon_{F} \approx 2.09 \mu \sec A\left(n / n_{0}\right)^{-2 / 3}$ and takes on the form [10

$$
\tau_{\text {form }} \sim \frac{\tau_{F e r m i}}{2 \pi}\left[\frac{T_{F}}{T_{C}}\right]^{2} \approx \frac{10}{2 \pi} \tau_{F e r m i} \exp (0.8 r /|a|) .
$$

The requirement that the superfluid forms before the gas is depleted, $\tau_{\text {form }}<\tau_{L}$, constrains the scattering length according to $|a| / r>0.8 /\left[\ln (2 \pi / 10)+\ln \left(\tau_{L} / \tau_{\text {Fermi }}\right)\right] \approx$ 
$0.35 / \log _{10}\left(\tau_{L} / \tau_{\text {Fermi }}\right)$ (assuming $\tau_{L}>>10^{2} \tau_{\text {Fermi }}$ ). For typical values, $\tau_{\text {Fermi }} \sim 10-100 \mu$ sec and $\tau_{L} \sim 100$ seconds, $\log _{10}\left(\tau_{L} / \tau_{\text {Fermi }}\right) \sim 6-7$, we find that the scattering length has to exceed $5 \%$ of the average inter-particle distance, $|a| / r \geq 0.05-0.06$. Atom gas Cooperpairing thus requires an effective interaction that is unusually strong for neutral atoms. Such interaction can be realized by using atoms that form a virtual state in the binary scattering, or by using external fields that alter the atom-atom interactions. On the other hand, when the magnitude of the negative scattering length exceeds $|a|>0.48 r$ (corresponding to $k_{F}|a|>3 \pi / 5,[8]$ ), the pressure turns negative and the gas collapses. This requirement of mechanical stability limits the critical Cooper-pairing temperature to $T_{c}<T_{c, \max } \approx 0.1 T_{F}$.

Hole heating Next, we illustrate the heating caused by the loss-induced creation of fermiholes. We consider a homogeneous fermion mixture that is 'normal' (i.e. not superfluid) and initially at zero temperature. The single particle distributions are filled fermi-spheres in momentum space, corresponding to occupation numbers $n_{j, \mathbf{k}}=1(j=1,2)$ if $k \leq k_{F}$, $n_{j, \mathbf{k}}=0$ if $k>k_{F}$. The loss processes 'perforate' the fermi-spheres, creating holes that bring the system into a state that is not the ground-state (which has filled fermi-spheres of reduced radius). In due course, the system relaxes to its thermal equilibrium at finite temperature. The scattering processes that relax the system are interesting in their own right. A hole in Fermi-sphere 1 is filled by a particle of initially higher energy. This fermion 1 can change its momentum by interacting with a fermion 2 that is thereby promoted to an energy above the Fermi-level. Such particle-hole scattering, akin to traditional Auger scattering, continuously produces particles with energies up to $2 \epsilon_{F}$, energies that are anomalously high from the perspective of a thermal distribution. The resulting 'high-energy' fermions are scattered to lower energy states (or 'cooled').

The importance of hole heating follows from the rate of temperature increase which depends on the specific heat per particle, $c_{V}=\left(\pi^{2} / 2\right) k_{B}\left[k_{B} T / \epsilon_{F}\right]$ and on the rate with which particles are removed,

$$
\dot{n}_{j, \mathbf{k}}=-\frac{1}{\tau_{L}} n_{j, \mathbf{k}}
$$


where the system's lifetime, $\tau_{L}$, can depend on the density $\left(\tau_{L}^{-1}=\gamma, \beta n, \alpha n^{2}\right.$ for background, two- and three-body loss respectively) [13]. The removal of a single particle of momentum k lowers the energy of $N$ fermions at temperature $T, E(N, T)$, by its kinetic energy but increases the energy relative to that of the remaining particles, $N-1$ in total, at the same temperature,

$$
\begin{aligned}
E^{\prime} & =E(N, T)-\frac{\hbar^{2} k^{2}}{2 m} \\
& \approx E(N-1, T)+\left[\epsilon_{F}-\frac{\hbar^{2} k^{2}}{2 m}\right],
\end{aligned}
$$

where we assumed $T<<T_{F}$ so that the chemical potential is approximately equal to the Fermi-energy: $E(N, T)-E(N-1, T) \approx \epsilon_{F}$. Therefore, upon removal of a particle of momentum $\mathbf{k}$, the system 'gains' $\left[\epsilon_{F}-\hbar^{2} k^{2} / 2 m\right]$ in excess to the energy of the remaining particles at the same temperature. The probability to create a hole in the $(j, \mathbf{k})$-state after a short time interval $\Delta t$ is equal to $-\Delta t \dot{n}_{j, \mathbf{k}}=\frac{\Delta t}{\tau_{L}} n_{j, \mathbf{k}}$. The total excess energy gathered by the system during $\Delta t$ is the $(j, \mathbf{k})$ - excess energy multiplied by the probability for hole creation, summed over all $(j, \mathbf{k})$-states:

$$
\begin{aligned}
\Delta E & =\left(\frac{\Delta t}{\tau_{L}}\right) \sum_{j, \mathbf{k}} n_{j, \mathbf{k}}\left[\epsilon_{F}-\frac{\hbar^{2} k^{2}}{2 m}\right] \\
& =\left(\frac{\Delta t}{\tau_{L}}\right) \frac{4}{5} N \epsilon_{F} .
\end{aligned}
$$

The corresponding temperature increase $\Delta T=\Delta E /\left[2 N c_{V}\right]$ gives a rate, $\dot{T}=\Delta T / \Delta t$, equal to

$$
\dot{T}=\frac{\Delta E}{\Delta t C_{V}}=\left(\frac{4}{5 \pi^{2}}\right) \frac{T_{F}^{2}}{\tau_{L} T}
$$

The solution to Eq.(6), parametrized by the initial temperature $T_{0}$ and the 'temperature doubling time', $\tau_{2}$,

$$
T=T_{0} \sqrt{1+\frac{3 t}{\tau_{2}}} ; \tau_{2}=\left(\frac{15 \pi^{2}}{8}\right)\left[\frac{T_{0}}{T_{F}}\right]^{2} \tau_{L}
$$

shows that the system doubles its temperature in a time that is proportional to the square of the Fermi to initial temperature ratio, $\tau_{2} \sim 20\left[T_{0} / T_{F}\right]^{2} \tau_{L}$. Thus, fermions brought to $1 \%$ of their Fermi-temperature double their temperature in $\sim 0.2 \%$ of their lifetime. 
The above derivation assumes a constant loss-rate, whereas a change in the density alters this rate on a long enough time scale. Also, the use of the specific heat, a statistical quantity, may give the impression that the validity of the above derivation requires the system to remain in thermal equilibrium. To address both issues we determine the temperature from the observation that the energy-per-particle, $e$, of a homogeneous fermion gas or fermion gas mixture, is not affected by the particle loss (3), or by the subsequent scattering that brings the system to thermal equilibrium. In the Fermi-degenerate regime, we know that $e(N, T) \approx(3 / 5) \epsilon_{F}(N)+\left(\pi^{2} / 4\right)\left[\left(k_{B}^{2} T^{2}\right) / \epsilon_{F}(N)\right]$, correct up to order $\left[T / T_{F}\right]^{2}$. Equating $e(N, T)$ to its initial value $e\left(N_{0}, T_{0}\right)$, we solve for $T$. Denoting the initial Fermi-temperature by $T_{F, 0}$, using that $\left[\epsilon_{F}\left(N_{0}\right) / \epsilon_{F}(N)\right]=\left[N_{0} / N\right]^{2 / 3}$, and $\left[T_{F} / T_{F, 0}\right]=\left[N / N_{0}\right]^{2 / 3}$, we obtain

$$
\begin{aligned}
& \frac{T}{T_{F}}=\left(\frac{T_{0}}{T_{F, 0}}\right)\left(\frac{N}{N_{0}}\right)^{-1 / 3} \times \\
& \sqrt{1+\frac{12}{5 \pi^{2}}\left[\frac{T_{F, 0}}{T_{0}}\right]^{2}\left[1-\left(\frac{N}{N_{0}}\right)^{2 / 3}\right]} .
\end{aligned}
$$

To obtain a time-dependent expression, we substitute the fraction of remaining particles by $N(t) / N_{0}=\exp \left(-t / \tau_{L}\right),\left[1+t / \tau_{L}\right]^{-1},\left[1+2 t / \tau_{L}\right]^{-1 / 2}$ for loss that is predominantly caused by background, two- and three-body scattering respectively. In Fig.(1), we graph the time-dependent temperature to Fermi-temperature ratio for $T_{0}=0.01 T_{F}$. The short-time behavior, $t<0.1 \tau_{L}$, is indeed well described by Eq. (7). On the scale of the graph $\left(t \sim \tau_{L}, T \sim 0.1 T_{F}\right)$ the plot is 'universal' in the sense that any temperature curve of lower initial value is indistinguishable from that shown (described by the $T_{0} \rightarrow 0$-limit, $\left.T \rightarrow T_{F, 0} \sqrt{\left(12 / 5 \pi^{2}\right)\left[\left(N / N_{0}\right)^{-2 / 3}-1\right]}\right)$. After $t \approx 0.1 \tau_{L}$, the temperature increase slows down and $T$ reaches $25 \%-30 \%$ of the Fermi-temperature by 'middle age', $t \sim 0.5 \tau_{L}$. At later times, the fermion system becomes classical (invalidating the above expression of $e(N, T)$ ) and $\lim _{t \rightarrow \infty} T(t)=0.4 T_{F, 0}$, which follows from $e_{\text {classical }}=(3 / 2) k_{B} T \approx(3 / 5) \epsilon_{F}\left(N_{0}\right)$. The above result does not depend on thermal equilibrium, provided we interpret temperature as a measure of the difference of the system's energy with its ground state value.

Superfluid formation and heating as competing effects Regardless of the initial temper- 
ature, the fermions heat up to at least $10 \%$ of the Fermi-temperature after only $7 \%$ of the system's lifetime. The temperature $T=0.1 T_{F}$ is the highest possible critical temperature for fermion superfluidity, $T_{c, \max } \approx 0.1 T_{F}$, therefore, the maximal time the fermion superfluid has to form is $0.07 \tau_{L}$, not $\tau_{L}$. It may, in fact, be difficult to realize $T_{c, \text { max }}$ and we consider the more general constraints on the interaction from the requirement of forming the Cooper-paired superfluid before the system heats up above the critical temperature:

$$
T\left(t=\tau_{\text {form }}\right)<T_{c}
$$

Because the superfluid formation time cannot exceed $0.07 \tau_{L}, T_{0} \sqrt{1+3 t / \tau_{2}}$ (7) adequately describes the temperature, and we find from (9)

$$
\tau_{2}>\frac{3 \tau_{\text {form }}}{\left(T_{c} / T_{0}\right)^{2}-1}
$$

Substituting $\tau_{2}$ and $\tau_{\text {form }}$ in terms of $\tau_{L}\left(\right.$ (7) and $\tau_{\text {Fermi }}(2)$, the inequality (10) yields

$$
\tau_{L}>\left(\frac{4}{5 \pi^{3}}\right) \frac{\left(T_{F} / T_{c}\right)^{4}}{1-\left(T_{0} / T_{c}\right)^{2}} \tau_{F e r m i} .
$$

If the experimentalist succeeds in bringing the initial temperature of the system significantly below the critical temperature (e.g. $T_{0} \leq 0.3 T_{c}$ ), then the denominator in (11) can be replaced by 1 and the condition becomes independent of $T_{0}$. Assuming $T_{0} \leq 0.3 T_{c}$ and substituting $T_{c} / T_{F}$ by $0.3 \exp (-0.4 r /|a|)$, we finally obtain

$$
\begin{aligned}
\frac{|a|}{r}> & \frac{0.4}{\ln \left[0.3 /\left(4 / 5 \pi^{3}\right)^{1 / 4}\right]+(1 / 4) \ln \left(\tau_{L} / \tau_{\text {Fermi }}\right)} \\
& \approx \frac{0.69}{\log _{10}\left(\tau_{L} / \tau_{\text {Fermi }}\right)}
\end{aligned}
$$

where the last approximation is valid if $\tau_{L} \geq 10^{4} \tau_{\text {Fermi }}$. Note that the minimal value of the dimensionless interaction strength $|a| / r$ is approximately twice as large as from the condition $\tau_{\text {form }}>\tau_{L}$. Thus, if $\tau_{L} / \tau_{\text {Fermi }} \sim 10^{6}-10^{7}$, the minimal value the negative scattering length has to take on to form Cooper-pairs is, $|a| / r>0.125-0.106$.

Equation (9) tacitly assumes that Fermi-holes relax faster than the superfluid can form, an assumption we now justify by calculating the lifetime of a hole. Specifically, we consider 
a fermion 1 hole in the center of the Fermi-sphere $(1, \mathbf{k}=0)$, and we determine its inverse lifetime $\tau_{h, \mathbf{k}=0}^{-1}$ in a Fermi-Golden rule calculation as the rate at which the hole "fills up" by scattering processes $(1, \mathbf{q})+(2, \mathbf{p}) \rightarrow(1, \mathbf{k}=0)+(2, \mathbf{p}+\mathbf{q})$ :

$$
\tau_{h, \mathbf{k}=0}^{-1}=\left(\frac{2 \pi}{\hbar}\right)\left[\frac{4 \pi \hbar^{2}}{m a \Omega}\right]^{2} \sum_{\mathbf{p}, \mathbf{q}} n_{1, \mathbf{q}} n_{2, \mathbf{p}}\left(1-n_{2, \mathbf{p}+\mathbf{q}}\right) \delta\left(E_{\text {initial }}-E_{\text {final }}\right)
$$

where $\Omega$ denotes the volume in box normalization. With the $(1, \mathbf{k}=0)$ final state, the energy density reduces to $\delta\left(E_{\text {initial }}-E_{\text {final }}\right) \rightarrow\left(m / \hbar^{2} p q\right) \delta(\cos \theta)$, where $\theta$ denotes the angle between $\mathbf{q}$ and $\mathbf{p}$. The (q, p)-integral with zero-temperature Fermi Dirac occupation numbers yields $\tau_{h, \mathbf{k}=0}^{-1}=\left(4 \pi a^{2}\right) n\left(\frac{3 \hbar k_{F}}{4 m}\right)$. Remarkably, $\tau_{h, \mathbf{k}=0}^{-1}$ equals the classical estimate for the collision rate of a zero velocity particle in the presence of the distinguishable particles, $\tau_{\text {coll }}^{-1}$ where $\tau_{\text {coll }}=[\langle\sigma n v\rangle]^{-1}=0.207 \tau_{\text {Fermi }}(r /|a|)^{2}$. Replacing the Pauli-blocking factor $\left(1-n_{2, \mathbf{p}+\mathbf{q}}\right)$ in (13) by 1 reduces the lifetime to half its value. Therefore, whereas Pauli-blocking increases the lifetime of holes and particles drastically near the Fermi-surface, its effect on the hole lifetime is moderate near the center of the Fermi-spehere where $\tau_{h, \mathbf{k}} \sim \tau_{\text {coll }}$, which is also the time scale on which the high energy $(2, \mathbf{p}+\mathbf{q})$-fermions scatter. Finally, $\tau_{\text {form }} / \tau_{\text {coll }} \approx$ $7.7(|a| / r)^{2} \exp \left(0.8 \frac{r}{|a|}\right)$, so that at its minimum $(|a|=0.4 r) \tau_{\text {form }} / \tau_{\text {coll }} \approx 9.5$ and $\tau_{\text {form }} / \tau_{\text {coll }}$ quickly increases as $|a|$ is reduced. The implication is that that there is a region inside the Fermi-sphere where holes are short-lived on the time scale of superfluid formation. The decay of those holes produces 'high energy' fermions that start heating the system while the superfluid forms.

While the treatment (9)-(12) certainly simplifies much of the intricate dynamics, we can trust the accuracy of the final constraint (12) to at least 15\%. Consider, for instance, the possibility that the non-equilibrium nature of the formation process, or the change of $c_{V}$ as the superfluid is formed, slows down the heating or favors the superfluid creation so as to weaken the condition (9) effectively by as much as one order of magnitude $T\left(t=\tau_{\text {form }} / 10\right)<$ $T_{c}$. This amounts to multiplying $\tau_{L}$ by a factor of 10 , giving, under the same conditions as above a range of minimal values $|a| / r \sim 0.106-0.092$.

Finally, we claim that our estimates are lower bounds. In a spherically symmetric 
harmonic trap the same reasoning as for the homogeneous mixture leads to the following time-dependence for the temperature as a result of background scattering: $T / T_{F}=$ $\left[T_{0} / T_{F, 0}\right]\left(N / N_{0}\right)^{-1 / 6} \sqrt{1+\left(3 / \pi^{2}\right)\left(T_{F, 0} / T_{0}\right)^{2}\left[1-\left(N / N_{0}\right)^{1 / 3}\right]}$ [14], with $N / N_{0}=\exp \left(-t / \tau_{L}\right)$. On the other hand, two- and three-body rates are highest in the high-density middle of the trap where the energy per particle is lowest. Thus, lower energy atoms are removed preferentially [15], giving a temperature that increases more rapidly than in the homogeneous system. Secondly, rates for some recombination processes are sensitive to the value of the scattering length. A large value of $|a|$ can imply a shorter lifetime, in which case $\tau_{L}$ in (12) really depends on $|a|$ [16], [17].

In conclusion, we have determined how a metastable degenerate fermion gas heats from the hole creation caused by the loss processes. The heating rate is particularly significant at ultra-cold temperatures, with a temperature doubling time, $\tau_{2} \sim 20\left[T_{0} / T_{F}\right]^{2} \tau_{L}$. On longer time scales $t \sim 0.5 \tau_{L}$, the fermion system heats up to $T \geq 0.25 T_{F}$. For the quest of atomtrap fermion superfluidity, this heating competes with the formation of the superfluid and we derive a minimal value for the unlike-fermion scattering length to observe superfluid formation in a gas mixture of unlike fermion atoms. In contrast to the simple mixture of fermion atoms, sympathetic cooling can provide a third (bosonic) atom species that continues to absorb the energy released by hole creation. Given the importance of the hole-heating mechanism, such continuous cooling may be helpful to reach fermion superfluidity.

In a question at a Los Alamos colloquium, Albert Pecheck remarked upon the problem of Fermi-holes. The fact that this problem did not appear to have been addressed, motivated this work. The author also gratefully acknowledges discussions on this subject with Peter Milonni, Kyoko Furuya, Randy Hulet, Xinxin Zhao and Michael Di Rosa. 


\section{REFERENCES}

[1] M. H. Anderson, et al., Science 269, 198 (1995); C. C. Bradley, et al., Phys. Rev. Lett. 75, 1687 (1995); K. B. Davis, et al., Phys. Rev. Lett. 75, 3969 (1995).

[2] For a recent review see "Superfluids and superfluid mixtures in atom traps", Eddy Timmermans, Contemporary Physics, 42, 1 (2001), (Taylor \& Francis) and references therein.

[3] A particularly intriguing prospect is the possibility of observing a BCS-BEC cross-over, see A. J. Leggett, in Modern Trends in the Theory of Condensed Matter, eds. A. Pekalski and J. Przystawa (Springer-Verlag, Berlin, 1980), C. A. R., Sa de Melo, Mohit Randeria, and Jan R. Engelbrecht, Phys. Rev. Lett., 71, 3202 (1993).

[4] B. DeMarco, D. S. Jin, Science, 285, 1703 (1999).

[5] A. G. Truscott et al., 'Observation of Fermi Pressure in a Gas of Trapped Atoms', Science, 291, 2570 (2001).

[6] F. Schreck et al., cond-mat/0011291 (2000).

[7] M. S. Holland, B. D. De Marco, and D. S. Jin, Phys. Rev. A 61, 53610 (2000).

[8] H.T.C. Stoof et al., Phys. Rev. Lett. 76, 10 (1996); and M. Houbiers et al., Phys. Rev. A 56, 4864 (1997).

[9] The mean-field result for the prefactor in the expression for $T_{c}, 0.6$ [3], is reduced by $50 \%$ when the fluctuations are included in the calculation: L. P. Gorkov, and T. K. Melik-Barkhudarov, Soviet Physics JETP, 13, 1018 (1961); H. Heiselberg et al., Phys. Rev. Lett., 85, 2418 (2000).

[10] M Houbiers, H. T. C. Stoof, Phys. Rev. A, 59, 1556 (1999).

[11] Barring unusual circumstances, the lifetime $\tau_{L}$ due to inelastic scattering of the particle is effectively energy independent. 
[12] E. R. I. Abraham et al., Phys. Rev. A, 55, R3299 (1997).

[13] As Fermi-statistics prohibits three fermions from residing simultaneously in each other's proximity, three-body inelastic scattering will be suppressed in a degenerate two fermion mixture: Chris Greene, private communications and Randy Hulet, private communications. However, an intermediate state molecule formed in the Feshbach resonance can undergo vibrational relaxation when encountering a third fermion some time after its formation. Off-resonance, such loss-rate can take on the form of three-body loss, E. Timmermans et al., Phys. Rep. 315, 199 (1999).

[14] A lengthy but straightforward calculation gives the following expression for the energy per particle in a spherically symmetric harmonic trap of degenerate fermion particles:e $=$ $(3 / 4) \epsilon_{F}\left[1+\left(T / T_{F}\right)^{2} \pi^{2} / 3\right]$ where $\epsilon_{F}$ is the Fermi-energy, $\epsilon_{F}=\hbar \omega_{T}(6 N)^{1 / 3}$, and where $N$ denotes the total number of fermions and $\omega_{T}$ the trap frequency.

[15] C. A. Sackett et al., Phys. Rev. Lett., 82, 876 (1999).

[16] P. O. Fedichev et al., Phys. Rev. Lett., 77, 2921 (1996).

[17] B. D. Esry et al., Phys. Rev. Lett., 83, 1751 (1999). 
Fig. 1 Plot illustrating the loss-induced heating of the fermion mixture. For a homogeneous fermion gas mixture with initial temperature of $T=0.01 T_{F}$, we plot the ratio of temperature to fermi-temperature as a function of time for loss processes that are predominantly background (solid line), two-body (dashed line) and three-body (long-dashed line) processes [13]. The thin line curve shows $T / T_{F}$ for the short time temperature approximation, $T=T_{0} \sqrt{1+3 t / \tau_{2}}$ in the case of background scattering. 


\section{Fermi hole heating}

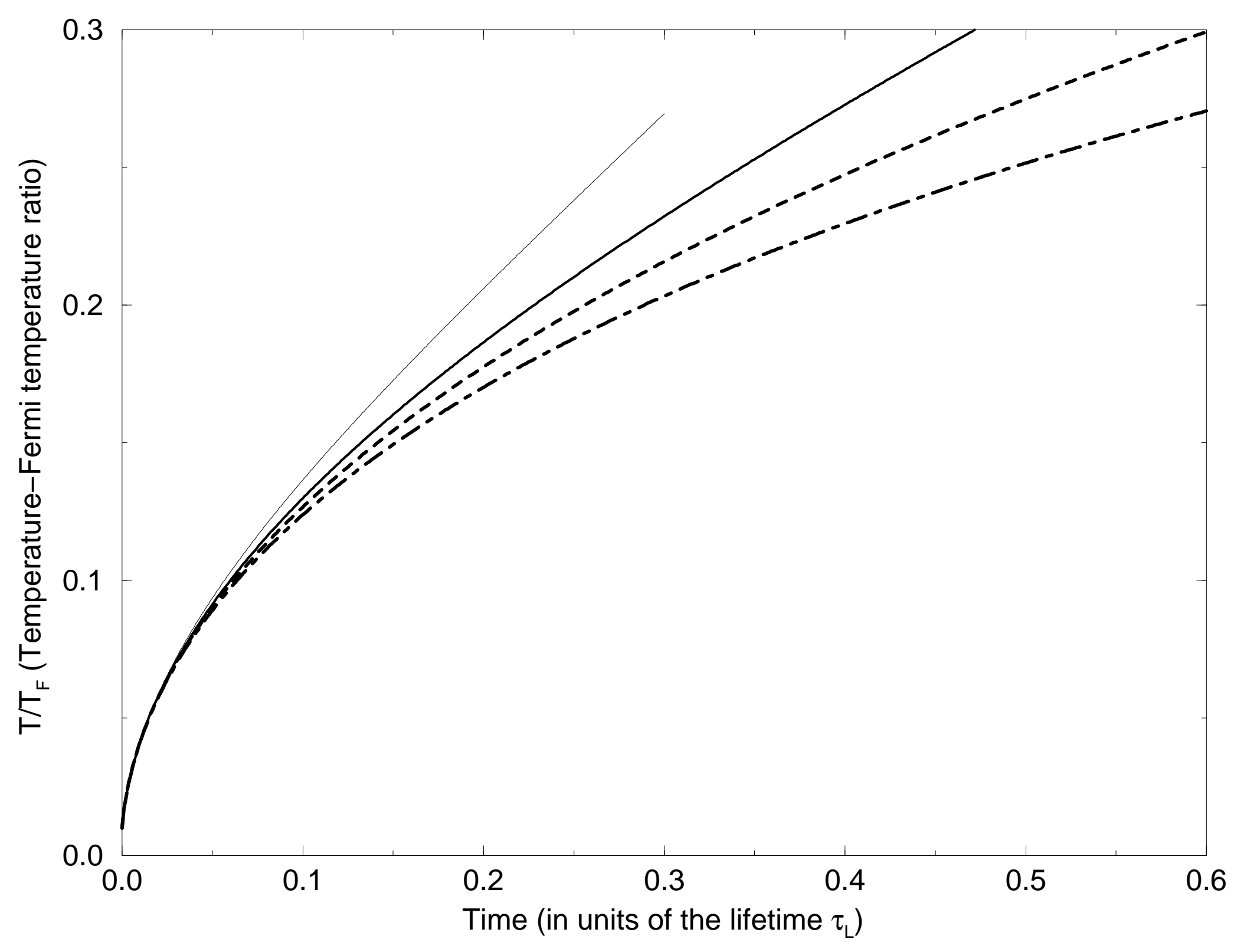

